\title{
Instance-level Accuracy versus Bag-level Accuracy in Multi-Instance Learning
}

\author{
Gitte Vanwinckelen • Vinicius Tragante \\ do $\mathrm{O}$ • Daan Fierens · Hendrik Blockeel
}

Received: date / Accepted: date

\begin{abstract}
In multi-instance learning, instances are organized into bags, and a bag is labeled positive if it contains at least one positive instance, and negative otherwise; the labels of the individual instances are not given. The task is to learn a classifier from this limited information. While the original task description involved learning an instance classifier, in the literature the task is often interpreted as learning a bag classifier. Depending on which of these two interpretations is used, it is more natural to evaluate classifiers according to how well they predict, respectively, instance labels or bag labels. In the literature, however, the two interpretations are often mixed, or the intended interpretation is left implicit. In this paper, we investigate the difference between bag-level and instance-level accuracy, both analytically and empirically. We show that there is a substantial difference between these two, and better performance on one does not necessarily imply better performance on the other. It is therefore useful to clearly distinguish the two settings, and always use the evaluation criterion most relevant for the task at hand. We show experimentally that the same conclusions hold for Area Under the ROC curve.
\end{abstract}

Keywords Classification · Multi-Instance Learning

Gitte Vanwinckelen · Daan Fierens · Hendrik Blockeel

KU Leuven, Department of Computer Science, Celestijnenlaan 200A, 3001 Leuven, Belgium, E-mail: firstname.lastname@cs.kuleuven.be, fierens.daan@gmail.com

Vinicius Tragante do O

Department of Cardiology, Division of Heart and Lungs, University Medical Center Utrecht, Utrecht, The Netherlands,

E-mail: v.tragante@umcutrecht.nl

Department of Medical Genetics, Division of Biomedical Genetics, University Medical Center Utrecht, Utrecht, The Netherlands 


\section{Introduction}

In their seminal paper on multi-instance learning, Dietterich et al (1997) define the multi-instance learning task as follows. We are given a set of bags of instances. Each instance has an unknown label (positive or negative). Each bag has a known label, which is positive if and only if at least one instance in the bag is positive, and negative otherwise. The task is to learn, from this information, a classifier that can predict the label of instances, and hence also of bags.

Dietterich et al.'s article has given rise to a variety of learning approaches, as well as some theoretical work on the problem. Although many papers refer to Dietterich et al's problem definition, the term "multi-instance learning" has gradually become used in a broader sense, namely, any kind of learning in a context where a single example is a bag of instances. The requirement that with each instance a label is associated, and the label of a bag is positive if and only if it contains at least one positive instance, is then dropped. Some authors refer to this broader setting as "generalized multi-instance learning" as opposed to Dietterich et al.'s "standard multi-instance learning". In this paper, when we refer to multi-instance learning, we refer to the "standard" setting.

Within standard multi-instance learning, there are two interpretations of the problem; one is that the task is to learn a classifier for bags, the other that the task is to learn a classifier for single instances. In the standard setting, these two are equivalent, so one could argue it does not matter which interpretation is used. But this is not entirely the case: as we will argue further on, it is possible that, among two classifiers, the one with best performance for bag classification has worst performance for instance classification, and vice versa.

Although Dietterich et al. explicitly stated that they wanted to learn an instance classifier, their evaluation is based on bag classification. They do not mention the reason for this, but at least two reasons are plausible. First, from a predictive point of view, bag classification performance is indeed relevant for the problem they focused on (classifying molecules as having the "musk" property or not), even if, from the point of view of knowledge discovery, the task is to identify the conditions $C$ under which an instance is positive (which makes instance classification performance more relevant).

The second reason is more pragmatic: in the Musk datasets, the true instance labels are not known, only the bag labels are. That is, the correct solution to the multi-instance learning problem is not known, and hence cannot be compared to. This contrasts with the normal evaluation procedures used in machine learning: there, the labels of test instances are not given to the learner, but they are known to the researcher, and used to evaluate the learner's performance. In multi-instance learning, when individual instance labels are not known, a comparably reliable evaluation procedure is not available. Evaluation based on bag classification may then be the best available alternative. 
Up till now, however, it has not been investigated how valid bag classification evaluation is, as an approximation to instance classification evaluation. That is, when model $\mathrm{A}$ is shown to have better bag classification performance on some dataset than model $\mathrm{B}$, is it reasonable to assume it will also have better instance classification performance? Many papers implicitly make this assumption.

In this paper, we evaluate that assumption for two performance measures: Accuracy and Area Under the ROC Curve (AUC). We show that instance classification accuracy is not a monotonic function of bag classification accuracy. When ranking multi-instance classifiers according to one or the other, the correlation between them is positive, but substantially lower than one. Our experiments show that the same holds for AUC. We quantify the probability of choosing a suboptimal model when bag classification performance is substituted for instance classification performance. We also discuss what this entails in practice for some published results. Our main conclusion is that research on multi-instance classification would benefit from datasets with known instance labels, allowing for more accurate evaluation.

This paper is structured as follows. We first review the basics of multiinstance learning and briefly discuss the mathematical relationship between instance- and bag-level classification performance (Section 2). Next, we discuss the literature on multi-instance learning and emphasize the different interpretations of the learning task being used, and the link with the two different evaluation settings (Section 3). This section primarily focuses on accuracy as a performance measure. Next, we empirically analyze the relationship between bag-level performance and instance-level performance for both accuracy and AUC (Section 4). Finally, we empirically assess the benefit of special-purpose multi-instance learners versus reduction to regular single-instance learning, and compare this to earlier results (Section 5).

This article is based on work previously presented at the Benelux Conference on Artificial Intelligence (Tragante do $\mathrm{O}$ et al, 2011). It extends the earlier work in a number of ways. First, it contains a more detailed review of the background and the multi-instance learning literature (Section 2 and 3). Second, it provides a more extensive empirical analysis of the relationship between instance-level and bag-level performance. Third, it studies the influence of the inductive bias of the learner on the relationship between instanceand bag-level performance (Section 4). Finally, it compares the performance of special-purpose multi-instance learners with that of single instance learners trained on data with one-sided label noise (Section 5).

\section{Multi-instance learning: Preliminaries}

\subsection{Definition and terminology}

Let $\mathcal{X}$ be the instance space and $\mathbb{B}=\{$ pos, neg $\}$ the binary set of class labels. Standard binary classification, which we here call the single-instance setting, 
can be defined as follows. We are given a dataset $D$ consisting of elements $\left(\mathbf{x}_{i}, f\left(\mathbf{x}_{i}\right)\right)$ with $\mathbf{x}_{i} \in \mathcal{X}$ an instance and $f\left(\mathbf{x}_{i}\right) \in \mathbb{B}$ its label according to an unknown function $f: \mathcal{X} \rightarrow \mathbb{B}$. The learning task is to find the function $f$.

The multi-instance learning setting is typically defined as follows (Dietterich et al, 1997). We are given a dataset that consists of bags $B_{i}$ of instances; the number of instances in a bag is variable. Each instance is described by a single vector $\mathbf{x}_{i j} \in \mathcal{X}$. An instance can be positive or negative, but the instance labels are not given. Instead, we are given bag labels, and we know that a bag is labeled positive if it contains at least one positive instance, and negative otherwise. From this information, we are to learn a function that can classify instances or bags.

The first interpretation of the task is that an instance classifier is learned. This corresponds to the following definition.

Definition 1 (Multi-instance learning, interpretation 1)

Given: a dataset $D$ consisting of elements $\left(B_{i}, F\left(B_{i}\right)\right)$ with $B_{i}$ a bag of instances $\left\{\mathbf{x}_{i 1}, \ldots, \mathbf{x}_{i n_{i}}\right\}$ and $F\left(B_{i}\right) \in \mathbb{B}$ its label; there exists an unknown function $f: \mathcal{X} \rightarrow \mathbb{B}$ for which it holds that

$$
F(B)=\left\{\begin{array}{l}
\text { pos if } \exists x \in B: f(x)=\text { pos } \\
\text { neg otherwise. }
\end{array}\right.
$$

Find: $f$.

The second interpretation of the task is identical except that now the bag labeling function $F$ is to be learned.

Definition 2 (Multi-instance learning, interpretation 2)

Given: the same information as in Definition 1.

Find: $F$.

Both definitions are used in the literature (we present an overview later on), and both are interpretations of what we call "standard" multi-instance learning.

As said, there is also "generalized" multi-instance learning (Zhang, 2009). Here, the task is to learn $F$, but contrary to standard multi-instance learning, Equation 1 need not hold, and the notion of an instance classifier $f$ may not even exist. For that reason, this paper is relevant only for standard multiinstance learning.

\subsection{Connection between $f$ and $F$}

Equation 1 shows that $f$ uniquely determines $F$. The converse is also true: given $F$, it must hold that

$$
f(x)=F(\{x\}) .
$$

This follows formally from Definition 1: Since Equation 1 holds for all bags, it holds also for singletons, and for a singleton $\{e\}$ we have $F(e)=p o s \leftrightarrow \exists x \in$ $B: f(x)=$ pos $\Leftrightarrow f(e)=$ pos. 
Let $\mathcal{B}$ be the function that transforms an instance classifier into an equivalent bag classifier; that is, $F=\mathcal{B}(f)$ if and only if Equation 1 holds. Similarly, let $\mathcal{I}$ be the function that transforms a bag classifier $F$ into an instance classifier $f$; that is, $f=\mathcal{I}(F)$ if and only if Equation 2 holds. In standard multi-instance learning, the transformations $\mathcal{I}$ and $\mathcal{B}$ are guaranteed to be each other's inverse: $\mathcal{I}(\mathcal{B}(f))=f$ and $\mathcal{B}(\mathcal{I}(F))=F$.

Equation 1 is well-known to all multi-instance learning researchers, but the fact that Equation 2 trivially follows from it does not seem to have been given much consideration. It has been used implicitly in some cases; for instance, Zhou et al (2005) propose an algorithm for detecting regions of interest in images (Citation-ROI) that is really an application of the above rule for a particular multi-instance learner (Citation-kNN), though not presented as such.

\subsection{Instance-level versus bag-level accuracy}

From now on, we distinguish the real target concepts underlying the learning problem, denoted $f$ and $F$ as before, from the actual classifiers constructed by a multi-instance learner when applied on a dataset, denoted $\hat{f}$ and $\hat{F}$. Some multi-instance learners return an instance classifier $\hat{f}$; for these, we define the corresponding bag classifier as $\hat{F}=\mathcal{B}(\hat{f})$. Other multi-instance classifiers return a bag classifier; for these, we define $\hat{f}=\mathcal{I}(\hat{F})$. For the real target concepts, we have $f=\mathcal{I}(F)$ and $F=\mathcal{B}(f)$ as before.

We call a classifier perfect when it has zero error. Any method that learns a perfect bag classifier $(\hat{F}=F)$ learns a perfect instance classifier $(\hat{f}=\mathcal{I}(\hat{F})=$ $\mathcal{I}(F)=f)$, and vice versa. However, when learning from data, we typically do not find perfect classifiers; the learned function $\hat{f}$ (or $\hat{F}$ ) only approximates $f$ (or $F$ ).

We can distinguish two accuracy measures for a classifier $(\hat{f}, \hat{F})$ on a given multi-instance dataset.

- Bag-level accuracy $a_{B}$ is the proportion of bags for which the predicted label equals the true label. It measures how well $\hat{F}$ approximates $F$.

- Instance-level accuracy $a_{I}$ is the proportion of instances for which the predicted label equals the true label. It measures how well $\hat{f}$ approximates $f$.

Given the one-to-one relationship between $F$ and $f$ (Equations 1 and 2), one might expect that there is a similar link between the corresponding accuracy measures, $a_{B}$ and $a_{I}$. This is not the case, as we show next.

2.4 Mathematical analysis of the relationship between bag-level and instance-level accuracy

Under the simplifying assumption that instances in a bag are independent from each other, we can analyze the relationship between $a_{I}$ and $a_{B}$ analyt- 
ically, showing that there is no one-to-one correspondence between them in this special case (and hence, not in the general case either).

Specifically, we consider the following generative process: a random number of instances are drawn randomly and i.i.d., and put in a bag; the bag is labeled positive if and only if at least one of these instances is positive. The dataset consists of a number of bags, all generated in this way. The instance distribution and bag size distribution can be any distribution.

Accuracy is a weighted average of the true positive rate $T P$ (the proportion of positive cases that are predicted positive) and the true negative rate $T N$ (the proportion of negative cases predicted negative). Let $T P_{I}$ and $T N_{I}$ be the instance-level true positive/negative rate of $\hat{f}$ over the whole instance population. The expected instance-level accuracy of $\hat{f}$ on a set of $N_{I}$ instances, among which $p$ positives and $n$ negatives, is then

$$
a_{I}=\frac{p \cdot T P_{I}+n \cdot T N_{I}}{N_{I}} .
$$

The expected bag-level accuracy $a_{B}$ of the corresponding $\hat{F}$ on a set of $N_{B}$ bags, the elements of which are drawn i.i.d. from that population, is

$$
\begin{aligned}
a_{B} & =\frac{\sum_{B_{k} \in \mathbf{B}^{+}} a_{B}^{+}\left(B_{k}\right)+\sum_{B_{k} \in \mathbf{B}^{-}} a_{B}^{-}\left(B_{k}\right)}{N_{B}} \\
a_{B}^{-}\left(B_{k}\right) & =T N_{I}^{n_{k}}, \\
a_{B}^{+}\left(B_{k}\right) & =1-T N_{I}^{n_{k}}\left(1-T P_{I}\right)^{p_{k}}
\end{aligned}
$$

with $\mathbf{B}^{+}$and $\mathbf{B}^{-}$the set of positive and negative bags, respectively, and with $p_{k}$ and $n_{k}$ the number of positive/negative instances in bag $B_{k}$. Equation 4 writes the expected bag-level accuracy as an average of the expected classification accuracy of positive and negative bags. Equation 5 follows from the fact that a negative bag is predicted correctly if all its instances are correctly predicted negative. Equation 6 follows from the fact that a positive bag is predicted correctly if it is not predicted as negative; and a bag is predicted negative when all its negative members are correctly predicted negative (probability $T N_{I}^{n_{k}}$ ), and all its positive members are incorrectly predicted negative (probability $\left.\left(1-T P_{I}\right)^{p_{k}}\right)$.

Together, Equations 3 to 6 show that, for a given $a_{I}$, there is not a single corresponding value of $a_{B}$ (and vice versa): this value depends on the size of the bags $B_{k}$ and the instance class distribution, which are problem-dependent, and on $T P_{I}$ and $T N_{I}$, which are classifier-dependent. Hence, even on the same problem, different classifiers may exhibit a different relationship between baglevel and instance-level accuracy. Among two classifiers, one classifier may score best on one measure and worst on the other.

This is demonstrated by the following example. Consider applying two classifiers $C_{1}$ and $C_{2}$ on a dataset that is balanced on the bag level, with on the instance-level $1 / 7$ of the instances positive and $6 / 7$ negative. Classifier $C_{1}$ has $T N_{1}=0.2$ and $T P_{1}=0.9$, and $C_{2}$ has $T N_{2}=0.7$ and $T P_{2}=0.2$. 
Table 1 The instance- and bag-level accuracy of two classifiers, $C_{1} \quad(\mathrm{TN}=0.7, \mathrm{TP}=0.2)$ and $C_{2}(\mathrm{TN}=0.2, \mathrm{TP}=0.9)$, for two different bag configurations.

\begin{tabular}{c|ll}
\hline & $C_{1}$ & $C_{2}$ \\
\hline$a_{I}$ & $\frac{6}{7} \cdot 0.2+\frac{1}{7} \cdot 0.9=0.3$ & $\frac{6}{7} \cdot 0.7+\frac{1}{7} \cdot 0.2=0.63$ \\
$a_{B, \text { conf. } 1}$ & $\frac{1}{2}\left(0.7^{5}+1-0.7^{1}(1-0.2)^{1}\right)=0.30$ & $\frac{1}{2}\left(0.2^{5}+1-0.2^{1}(1-0.9)^{1}\right)=0.50$ \\
$a_{B, \text { conf.2 }}$ & $\frac{1}{2}\left(0.7^{1}+1-0.7^{5}(1-0.2)^{1}\right)=0.78$ & $\frac{1}{2}\left(0.2^{1}+1-0.2^{5}(1-0.9)^{1}\right)=0.60$ \\
\hline
\end{tabular}

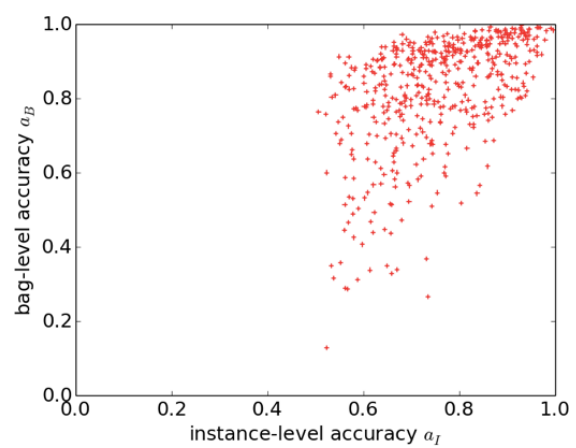

Fig. 1 Scatter plot showing for 500 problems with a randomly chosen $T P_{I}, T N_{I}$, class distribution and bag size distribution, the corresponding $a_{I}$ and $a_{B} \cdot a_{I}$ and $a_{B}$ correlate positively, but do not determine each other.

Using Equation 3, we find that the instance-level accuracy of the two classifiers is respectively $a_{I, 1}=0.3$ and $a_{I, 2}=0.63$. We create two different bag configurations from these instances. In the first configuration, negative bags contain five instances and positive bags contain one positive and one negative instance. In the second configuration, negative bags contain a single instance, and positive bags contain five negative and one positive instance. Applying Equations 3 to 6 for both bag configurations, Table 1 shows that depending on the configuration, either $a_{B, 1}$ is larger than $a_{B, 2}$ or vice versa.

The above also shows that $a_{I}$ is in a sense more 'stable' than $a_{B}$. If a model is found to have a particular $a_{B}$ on some test set, it may actually achieve a significantly different bag accuracy when predicting labels of bags that are significantly larger or smaller. (As an extreme example, if in a test set all bags are singletons, $a_{B}$ becomes equal to $a_{I}$ ). Note also that, given a class distribution and a bag size distribution, $T N_{I}$ and $T P_{I}$ determine the expected values of both $a_{I}$ and $a_{B}$. From this point of view, they are more fundamental than $a_{I}$ and $a_{B}$.

Figure 1 illustrates the relationship between $a_{I}$ and $a_{B}$ on some randomly generated datasets. It shows clearly that $a_{I}$ and $a_{B}$ correlate positively but do not determine each other.

Figure 2 shows how the class distribution affects $a_{I}$ and $a_{B}$, for a fixed $T N_{I}$ and $T P_{I}$. Depending on the value of $T N_{I}$ and $T P_{I}$, more positives in the instance population may increase both $a_{I}$ and $a_{B}$ (left), increase one but 

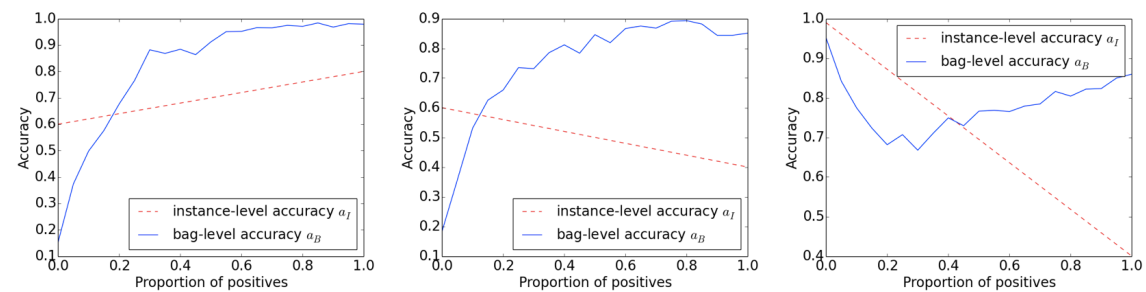

Fig. 2 Increasing the proportion of positive instances in the instance population from 0 to 1 may affect both $a_{I}$ and $a_{B}$ positively (left; $T P_{I}=0.8, T N_{I}=0.6$ ), affect one positively and the other negatively (middle: $T P_{I}=0.4, T N_{I}=0.6$ ), or have a more complicated effect (right; $\left.T P_{I}=0.4, T N_{I}=0.99\right)$.

decrease the other (middle), or have a more complicated effect (right; here, a maximal $a_{B}$ is obtained when $a_{I}$ is either very high or very low).

\section{Literature on (standard) multi-instance learning}

There is a large literature on multi-instance learning. This section is not meant to provide a complete overview, but merely to illustrate the points being made in this paper regarding variation in learning tasks and evaluation measures.

\subsection{Algorithms and applications}

Many algorithms for (standard) multi-instance learning have been developed. Perhaps the simplest approach is to label each instance with the label of its bag, then apply a standard learner to this dataset to obtain $\hat{f}$. Many instances will get a positive label in the training set even if they are really negative, but the opposite will not occur; thus, multi-instance learning reduces to learning from a dataset with one-sided class noise (Blum and Kalai, 1998). Such an approach was used as a "straw man" approach by Dietterich et al (1997), and shown not to work well, although Ray and Craven (2005) later showed that this may depend strongly on the application.

Many authors have proposed algorithms that explicitly address the peculiarities of the multi-instance setting, such as Axis-Parallel Rectangles (Dietterich et al, 1997), Diverse Density (Maron and Lozano-Pérez, 1998) and its extension EM-DD (Zhang and Goldman, 2001), neural networks (Ramon and De Raedt, 2000), the k-nearest neighbor algorithm Citation-kNN (Wang and Zucker, 2000), the decision tree algorithms ID3-MI (Zucker and Chevaleyre, 2001) and MITI (Blockeel et al, 2005), the rule learning algorithm RIPPERMI (Zucker and Chevaleyre, 2001), the SVM algorithms MI-SVM, mi-SVM (Andrews et al, 2003) and DD-SVM (Chen and Wang, 2004), the logistic regression algorithm MILR (Ray and Craven, 2005) and the ensemble algorithms MI-Ensemble (Zhou and Zhang, 2003) and MI-Boosting (Xu and Frank, 
2004). As the overview shows, many of these algorithms are inspired by singleinstance learning algorithms.

Applications of multi-instance learning include content-based image retrieval (Andrews et al, 2003; Maron and Ratan, 1998; Li et al, 2009; Fu et al, 2011; Zhou et al, 2005), music retrieval (Mandel and Ellis, 2008), protein family modeling (Tao et al, 2004) and medical applications (Fung et al, 2007) such as drug activity prediction (Dietterich et al, 1997). In image retrieval, many researchers have proposed the use of multi-instance learners to learn what the user's interest is. That is, given a series of pictures that are of interest to the user, presumably because they contain some object of interest (among other things), a multi-instance learner can be used to learn a function that can predict for new pictures whether they contain the object of interest or not. Some researchers consider as a separate task the detection of the regions of interest in these pictures ( $\mathrm{Li}$ et al, 2009; Fu et al, 2011; Zhou et al, 2005). Using our terminology, this boils down to identifying $f$.

\subsection{Learning task: Definition 1 versus Definition 2}

According to Definition 1, the goal is to learn an instance classifier, i.e., an approximation $\hat{f}$ to $f$. According to Definition 2, the goal is to learn a bag classifier, i.e., an approximation $\hat{F}$ to $F$. Both interpretations are used in the literature:

- In their seminal paper about multi-instance learning, Dietterich et al (1997) (p.34) state: "the goal of the machine learning algorithm will be to construct an approximation $\hat{g}$ to the internal function $g$ ", which in our notation is function $f$. This means that Dietterich et al. use Definition 1. This definition is also used by Blum and Kalai (1998), who note that multiinstance learning is a special case of learning from one-sided class noise. Some theoretical results regarding PAC learning axis-parallel rectangles in this setting (Long and Tan, 1998; Auer et al, 1998; Blum and Kalai, 1998) also use Definition 1, as do various other papers (e.g., Blockeel et al (2005); Maron and Lozano-Pérez (1998)).

- The Encyclopedia of Machine Learning (Ray et al, 2011) uses Definition 2. Many papers use this definition as well (e.g., Fung et al (2007); Maron and Ratan (1998); Wang and Zucker (2000); Zhang and Goldman (2001); Zucker and Chevaleyre (2001)).

It seems that the use of these two different interpretations of the learning task can be explained historically. When Dietterich et al (1997) introduced multi-instance learning, they considered Definition 1, i.e., learn $f$. In subsequent papers, there seems to be a shift in emphasis from the learning task to the data format. That is, many multi-instance papers clearly specify the format of the training data but leave it implicit whether the goal is to learn $f$ or $F$. Furthermore, multi-instance classifiers are typically evaluated in terms of bag-level performance, which relates to $F$ rather than $f$. This seems to 
have contributed to the second interpretation of the learning task (learn $F$ ) becoming more popular in recent papers.

\subsection{Performance measure: Bag-level versus instance-level}

Most papers evaluate classifiers according to bag level performance, regardless of whether Definition 1 or 2 is used. This is true for Dietterich et al, and for the large majority of cases we looked at (Dietterich et al, 1997; Maron and Lozano-Pérez, 1998; Zhang and Goldman, 2001; Wang and Zucker, 2000; Blockeel et al, 2005; Ramon and De Raedt, 2000; Zhou and Zhang, 2003; Xu and Frank, 2004; Zucker and Chevaleyre, 2001; Andrews et al, 2003; Ray and Craven, 2005; Tao et al, 2004; Fung et al, 2007). The reason for this is most likely that computing $a_{I}$ requires knowing the instance labels, which in most datasets are unknown. Exceptions are one paper on music information retrieval by Mandel and Ellis (2008), who explicitly state that they are interested only in $a_{I}$; a number of papers on content-based image retrieval (CBIR); and a recent theoretical study by Doran and Ray (2013).

In CBIR, two different tasks are often considered: (a) retrieving images that contain a particular type of object, and (b) identifying the region in the picture where that object occurs; this is also called ROI (region-of-interest) detection (Li et al, 2009; Fu et al, 2011; Zhou et al, 2005). Both learning problems can be seen as multi-instance learning, with images as bags and the different regions or segments as instances. Bag-level performance is then more relevant for task (a), instance-level performance for task (b), though for task (b) an alternative measure called success rate is often used: The classifier indicates for every bag predicted positive one instance that is most likely to be positive; the success rate is the proportion of positive bags for which a truly positive instance is indicated. Both forms of instance-level evaluation can only be automated if individual instance labels are available, which is often not the case; for this reason, several researchers (Fu et al, 2011; Shao et al, 2008) evaluate the ROI performance not quantitatively but only qualitatively, by manually inspecting detected ROIs for a small subset of all images.

Doran and Ray (2013) define a number of desirable but mutually exclusive properties of MI approaches based on support vector machines, and study how these affect performance; they find that the effect differs depending on whether one considers bag classification or instance classification. Their results are consistent with ours.

In summary, with a few exceptions as mentioned above, the large majority of the literature on multi-instance learning considers bag-level evaluation only. Given that in some applications instance-level performance is important too, this raises the question: what does bag-level performance tell us about instance level performance? Or, more concretely in the case of accuracy: to what extent does a high bag-level accuracy $a_{B}$ imply a high instance-level accuracy $a_{I}$ ? This question has not been addressed in the multi-instance literature, where $a_{I}$ and $a_{B}$ are often implicitly assumed highly correlated, or, on the contrary, 
no relationship whatsoever is assumed (viewing ROI detection and CBIR as separate tasks). We address this question in the next section, and do the same for AUC.

\section{Experimental analysis of the relationship}

As shown in Sections 2.4 and $3, a_{I}$ and $a_{B}$ cannot be expressed as a function of each other, let alone a monotonic function, and yet multi-instance classifiers are often evaluated based on bag-level performance, even when instance classification is desired. The question is then, how relevant such an evaluation is in practice. We investigate this empirically. While our theoretical analysis only covered accuracy as a performance measure, our empirical analysis also includes a comparison between instance-level $\mathrm{AUC}_{I}$ and bag-level $\mathrm{AUC}_{B}$. Multi-instance datasets are typically imbalanced on the instance-level, with many more negative instances than positive ones, so in these cases AUC may be a more appropriate performance measure than accuracy.

The first question we wish to address is: When comparing multiple classifiers on the same dataset, to what extent does a high bag-level accuracy imply a high instance-level accuracy, and vice versa? We will quantify this in two ways. First, the rank correlation $\rho\left(a_{I}, a_{B}\right)$ between the instance-level and bag-level accuracy of multiple classifiers on a given dataset can be computed. Second, the probability that a classifier scores higher on instance-level accuracy, given that it scores higher on bag-level accuracy, $P\left(a_{I}\left(C_{1}\right)>a_{I}\left(C_{2}\right) \mid a_{B}\left(C_{1}\right)>a_{B}\left(C_{2}\right)\right)$, with $C_{i}$ randomly chosen classifiers. While the first is a standard measurement for comparing rankings, the second is of practical importance: When we want to select, among two classifiers, the one with highest $a_{I}$, but instead choose the one with highest $a_{B}$ (or vice versa), what is the probability that we have chosen the correct one?

The comparison of multiple classifiers on the same dataset is of practical importance because it is frequently encountered in experimental evaluation. However, in order to understand the influence of the choice of the learner and the characteristics of the dataset on the relationship between bag- and instance-level performance, we ask a second question: When evaluating a particular learner on multiple datasets, to what extent does a high bag-level accuracy imply a high instance-level accuracy, and vice versa? We explore this question by constructing a scatter plot for each learner, plotting instance-level performance versus bag-level performance.

\subsection{Experimental setup}

\subsubsection{Datasets.}

In order to evaluate instance-level performance, we need multi-instance datasets that include not only the bag labels, as usual, but also the true instance labels. Most of the common benchmark multi-instance (MI) datasets, such as 
Musk (Dietterich et al, 1997), only contain bag labels and hence cannot be used for our purpose. MI datasets with known instance labels seem to be rare and are often not publicly available. Partly for that reason, we use a mix of realworld and semi-synthetic datasets. The latter are datasets that are based on a real-world single-instance dataset, but turned into a multi-instance dataset by grouping instances into bags and assigning labels to these bags according to the standard MI assumption.

Real-world: SIVAL. The SIVAL repository (Settles et al, 2008) ${ }^{1}$ is from the area of content-based image retrieval (CBIR). It contains 1500 images where each image contains one out of 25 different complex objects. The images are partitioned into 31 or 32 segments, i.e., the instances. An instance in this dataset consists of 30 features expressing color and texture information about the image segment and its four closest neighbors. The repository contains 25 multi-instance datasets where in turn each of the 25 objects is considered positive, while the other 24 objects are considered negative. Each of the 25 training sets consists of 60 positive and 60 negative bags. In positive bags, the percentage of positive instances varies from $3.1 \%$ to $90.6 \%$, with an average of $25.5 \%$.

Semi-synthetic: Text categorization. We use 20 MI text categorization datasets extracted by Settles et al (2008) from the 20 newsgroups corpus. ${ }^{2}$ Each dataset contains 100 bags and is balanced on the bag level. The size of the bags (number of instances) varies from 8 to 84 , with an average of 40 . A bag is a collection of short texts from the newsgroups. In positive bags, the percentage of positive instances varies from $2 \%$ to $7 \%$, with an average of $3.6 \%$. This is a high-dimensional dataset; each instance is characterized by 200 TFIDF features. The bags were artificially created by in turn considering one newsgroup as positive, while taking the other newsgroups as negative and i.i.d. sampling texts from the newsgroups such that around $3 \%$ of the texts in a bag are from the positive category.

Semi-synthetic UCI datasets. We have constructed some more semi-synthetic MI datasets from five source datasets taken from the UCI repository (Merz and Murphy, 1996): Adult (Kohavi, 1996), Pima Indians Diabetes (Smith et al, 1988), Spam (Cranor and LaMacchia, 1998), Tic-Tac-Toe (Aha, 1990) and Blood Transfusion Service Center (Yeh et al, 2009). We selected these datasets because they are imbalanced, which is useful for constructing MI datasets, since MI datasets that are balanced on the bag-level contain more negative than positive instances. All datasets are binary classification problems; we kept the labels unchanged, except for Tic-Tac-Toe, where we inverted the instance labels in order to have a majority of negatives.

We have constructed multiple MI datasets by choosing instances i.i.d. and grouping them into bags, controlling for bag size and positive/negative ratio in positive bags. We say that a $\mathrm{MI}$ dataset is in bag-configuration ' $X / Y$ ' if each bag in the dataset contains $Y$ instances, and each positive bag contains

1 Data available on http://pages.cs.wisc.edu/ bsettles/amil/.

2 Data available on http://lamda.nju.edu.cn/data_MItext.ashx. 
$X$ positive instances. For each source dataset, we use MI datasets in configurations $1 / 2,1 / 3$ and $2 / 3$; for the two largest (Adult and Spam) we additionally use $1 / 4,2 / 4,1 / 5,2 / 5,1 / 10$ and $2 / 10$. All MI datasets are balanced on the bag level: $50 \%$ of the bags is positive and $50 \%$ is negative. To construct a MI dataset in a particular bag-configuration, we randomly sampled the required number of positive instances and negative instances from the respective source dataset. The number of bags in each MI dataset is the highest possible number for which we can sample without replacement before exhausting the source dataset, except for Adult, where for computational reasons we retained only 1200 bags for each label, randomly chosen. Table 2 gives an overview of all the resulting MI datasets. The UCI datasets are complementary to the SIVAL and text datasets in the sense that they have large training set sizes, few features and relatively small bag sizes.

Table 2 Multi-instance dataset properties: Number of bags, number of instances per bag and percentage of positive instances per bag. The percentage of positive bags is $50 \%$ for all datasets. ('Ad.': Adult, 'TTT': Tic-Tac-Toe, 'Db.': Diabetes, 'Sp.': Spam, 'Tr.': Transfusion)

\begin{tabular}{cccc}
\hline Dataset & \#bags & $\begin{array}{c}\text { \#inst. } \\
\text { bag }\end{array}$ & $\begin{array}{c}\text { \%pos. } \\
\text { bag }\end{array}$ \\
\hline Ad. $1 / 2$ & 1200 & 2 & 50 \\
Ad. $1 / 3$ & 1200 & 3 & 33.3 \\
Ad. $1 / 4$ & 1200 & 4 & 25 \\
Ad. $1 / 5$ & 1200 & 5 & 20 \\
Ad. $1 / 10$ & 1200 & 10 & 10 \\
Ad. $2 / 3$ & 1200 & 3 & 66.6 \\
Ad. $2 / 4$ & 1200 & 4 & 50 \\
Ad. $2 / 5$ & 1200 & 5 & 40 \\
Ad. $2 / 10$ & 1200 & 10 & 20 \\
\hline TTT $1 / 2$ & 416 & 2 & 50 \\
TTT $1 / 3$ & 250 & 3 & 33.3 \\
TTT $2 / 3$ & 312 & 3 & 66.6 \\
\hline Db. $1 / 2$ & 332 & 2 & 50 \\
Db. $1 / 3$ & 198 & 3 & 33.3 \\
Db. $2 / 3$ & 248 & 3 & 66.6 \\
\hline
\end{tabular}

\begin{tabular}{cccc}
\hline Dataset & \#bags & $\begin{array}{c}\text { \#inst. } \\
\text { bag }\end{array}$ & $\begin{array}{c}\text { \%pos. } \\
\text { bag }\end{array}$ \\
\hline Sp. $1 / 2$ & 1858 & 2 & 50 \\
Sp. $1 / 3$ & 1114 & 3 & 33.3 \\
Sp. $1 / 4$ & 796 & 4 & 25 \\
Sp. $1 / 5$ & 618 & 5 & 20 \\
Sp. $1 / 10$ & 292 & 10 & 10 \\
Sp. $2 / 3$ & 1392 & 3 & 66.6 \\
Sp. $2 / 4$ & 928 & 4 & 50 \\
Sp. $2 / 5$ & 696 & 5 & 40 \\
Sp. $2 / 10$ & 308 & 10 & 20 \\
\hline Tr. $1 / 2$ & 356 & 2 & 50 \\
Tr. $1 / 3$ & 226 & 3 & 33.3 \\
Tr. $2 / 3$ & 178 & 3 & 66.6 \\
\hline SIVAL & 1500 & {$[31,32]$} & {$[3.1,90.6]$} \\
Text & 100 & {$[8,84]$} & {$[2,7]$} \\
\hline
\end{tabular}

\subsubsection{Learning algorithms.}

We have performed experiments with fourteen MI algorithms available in the Weka data mining tool (Witten and Frank, 2005): MIDD (Diverse Density) (Maron and Lozano-Pérez, 1998), MIEMDD (Expectation-Maximization Diverse Density) (Zhang and Goldman, 2001), MDD (Modified Diverse Density with collective assumption) (Dong, 2006), MISVM, which is a Weka implementation of the maximum pattern margin formulation mi-SVM by (Andrews et al, 2003), MIOptimalBall (Auer and Ortner, 2004), MILR (Logistic Regression) (Ray and Craven, 2005), logistic regression with the arithmetic mean model, referred to as MILRC from now on (Dong, 2006), MIRI (Bjerring and Frank, 


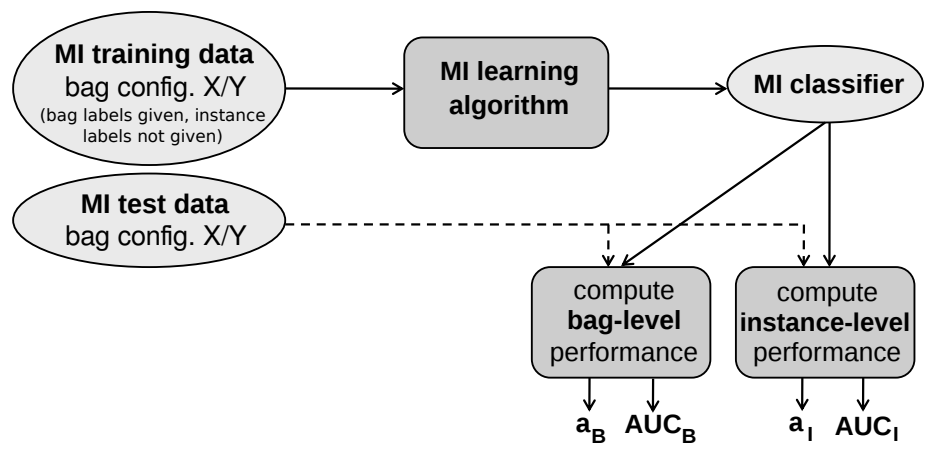

Fig. 3 Overview of the experimental setup.

2011), AdaBoost.M1 (Freund and Schapire, 1995) with a Multi Instance Tree Inducer (MITI) as base classifier (Blockeel et al, 2005), Citation-kNN (Wang and Zucker, 2000), TLD (Two-Level Distribution) (Xu, 2003), SimpleMI with the J48 classifier (Dong, 2006), MIWrapper with the J48 classifier (Frank and $\mathrm{Xu}, 2003)$, and MISMO, which is a Weka implementation of the normalized set kernel (NSK) by Gärtner et al (2002).

The parameter settings are as follows. MISMO uses the radial basis function $(\mathrm{RBF})$ kernel with $\gamma$ equal to 0.01 and the regularization parameter $\mathrm{C}$ equal to 1.0. MISVM uses the linear kernel with the regularization parameter $\mathrm{C}$ being 1.0. We also ran MISVM with the RBF kernel but found that this kernel did not lead to good performance on the text and SIVAL datasets. For MILR, the ridge coefficient equals $10^{-6}$. For Citation-kNN the number of citations and references both equal 5 .

Section 5 also compares a number of single instance algorithms to the corresponding multi-instance algorithms. Table 9 gives an overview of the corresponding algorithms. For logistic regression the ridge coefficient again equals $10^{-6}$. For Nearest neighbors the number of neighbors is 5 . We also used SMO with the radial basis function (RBF) kernel with $\gamma$ equal to 0.01 and $\mathrm{C}$ 1.0. Finally, for J48 the confidence factor equals 0.25 and the minimum number of instances per leaf is 2 .

Note that the algorithmic performances could be improved with parameter optimization. However, this would not influence the observed differences between instance-level and bag-level results. Due to computational limitations, we therefore limited the experiments to default parameter values.

\subsubsection{Setup.}

Figure 3 summarizes the experimental setup. We measure the bag-level performance and instance-level performance of each MI learning algorithm on each MI dataset with accuracy (respectively $a_{B}$ and $a_{I}$ ) and $\mathrm{AUC}\left(\mathrm{AUC}_{B}\right.$ and $\mathrm{AUC}_{I}$ ), using 10-fold bag-level cross-validation. 


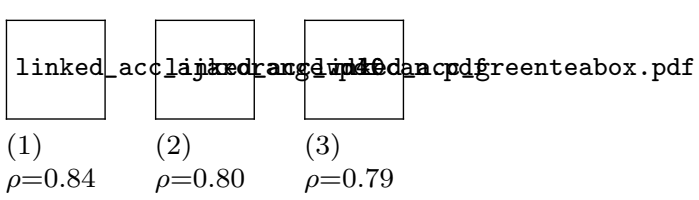

Fig. 4 Accuracy Rankings on SIVAL data: Differences between the ranking of all learning algorithms in terms of instance-level accuracy $a_{I}$ and bag-level accuracy $a_{B}$ for the SIVAL datasets. $\rho$ is the resulting rank correlation. (1) ajaxorange, (2) wd40can, (3) greenteabox.

\subsection{Results}

\subsubsection{Rank correlations.}

First, we have computed for each dataset the Spearman rank correlation $\rho\left(a_{I}, a_{B}\right)$ between instance-level and bag-level accuracy of the different methods:

$$
\rho\left(a_{I}, a_{B}\right)=\frac{\sum_{j}\left(r_{I}^{j}-\bar{r}_{I}\right)\left(r_{B}^{j}-\bar{r}_{B}\right)}{\sqrt{\sum_{j}\left(r_{I}^{j}-\bar{r}_{I}\right)^{2} \sum_{j}\left(r_{B}^{j}-\bar{r}_{B}\right)^{2}}},
$$

where $r_{I}^{j}$ is the rank of the $j$-th learning algorithm in terms of instance-level accuracy $(j=1 \ldots 10), \bar{r}_{I}$ is $\sum_{j=1}^{10} r_{I}^{j} / 14$, and $r_{B}^{j}$ and $\bar{r}_{B}$ are defined similarly in terms of bag-level accuracy. The Spearman correlation is 1 if $a_{I}$ and $a_{B}$ yield exactly the same ranking, -1 if they yield exactly opposite rankings, and 0 for independent rankings. The same formula can be used for AUC.

For each dataset category, SIVAL, text, and UCI, we present three representative results. For evaluation in terms of accuracy, these are shown respectively in Figures 4, 5 and 6. For each of the datasets, a plot visualizes the agreement between the instance-level and bag-level ranking: For the 14 MI learners, the $a_{I}$ and $a_{B}$ they resulted in (for that particular dataset) are ranked, and each $a_{I}$ is connected with the corresponding $a_{B}$. The extent to which the lines cross is a visual indication of the disagreement between the different measures. Overall, the results show that, while there is clearly some correlation between $a_{I}$ and $a_{B}$, it is relatively weak. It happens quite often that an algorithm performs well in terms of $a_{B}$ but poorly in terms of $a_{I}$, and vice versa (many crossing lines). The amount of disagreement depends on the type of dataset. Overall, the rank correlations are highest for the UCI datasets and lowest for the SIVAL datasets.

Figures 7, 8 and 9 show the results of the same analysis, but for AUC. Again the rank correlations are highest for the UCI datasets and lowest for the SIVAL datasets. For the UCI datasets, $\rho\left(\mathrm{AUC}_{I}, \mathrm{AUC}_{B}\right)$ is often close enough to one to consider $\mathrm{AUC}_{I}$ a good approximation of $\mathrm{AUC}_{B}$, and vice versa. For the text and SIVAL datasets this is not the case.

The results for the remaining datasets are similar and are available upon request to the authors. 


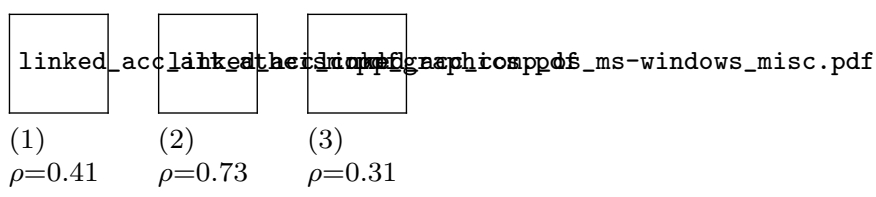

Fig. 5 Accuracy Rankings on text data: (1) alt atheism, (2) comp graphics, (3) comp os ms-windows misc.

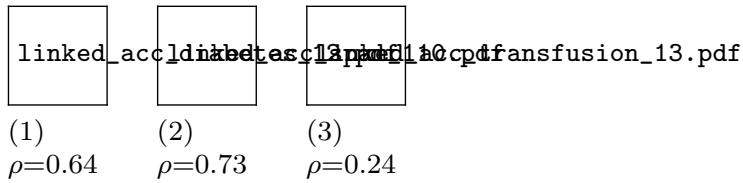

Fig. 6 Accuracy rankings on UCI data: (1) Diabetes 1/2, (2) Spam 1/10, (3) Transfusion $1 / 3$

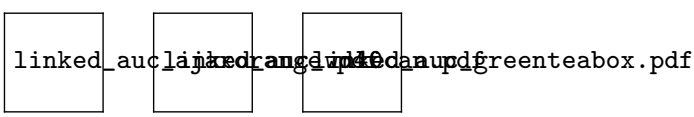
(1) $\rho=-$
(2) $\rho=-$
(3) $\rho=-$
0.41
0.56
0.43

Fig. 7 AUC Rankings on SIVAL data: Differences between the ranking of all learning algorithms in terms of instance-level $\mathrm{AUC}_{I}$ and bag-level $\mathrm{AUC}_{B}$ for the SIVAL datasets. $\rho$ is the resulting rank correlation. (1) ajaxorange, (2) wd40can, (3) greenteabox.

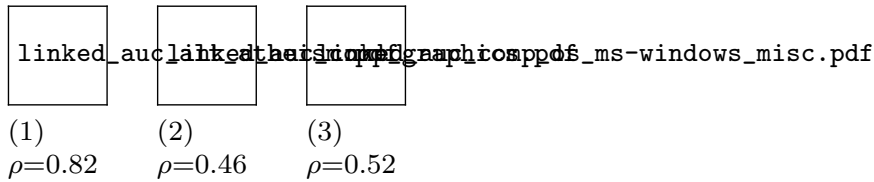

Fig. 8 AUC Rankings on text data: (1) alt atheism, (2) comp graphics, (3) comp os mswindows misc.

\subsubsection{Probabilistic analysis.}

As an alternative to the use of rank correlations, we also perform a probabilistic analysis. We compute a $95 \%$ Wilson confidence interval for $P\left(a_{I}\left(C_{1}\right)>\right.$ $\left.a_{I}\left(C_{2}\right) \mid a_{B}\left(C_{1}\right)>a_{B}\left(C_{2}\right)\right)$, i.e., the probability that classifier $C_{1}$ outperforms classifier $C_{2}$ in terms of instance-level accuracy, given that it outperforms it in terms of bag-level accuracy. We use $P_{I \mid B}$ as shorthand for this probability. Fol-

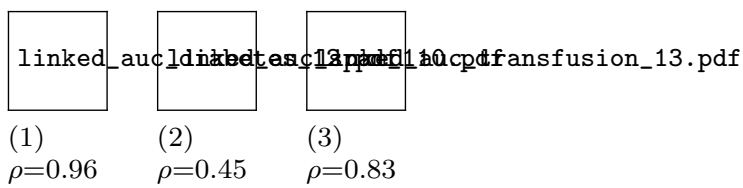

Fig. 9 AUC rankings on UCI data: (1) Diabetes 1/2, (2) Spam 1/10, (3) Transfusion 1/3. 
Table 3 Probabilistic analysis on SIVAL data for accuracy. 95\% confidence intervals for the probability that $a_{I}$ is higher, given that $a_{B}$ is higher $\left(\hat{P}_{I \mid B}\right)$.

\begin{tabular}{lc|lc}
\hline Dataset & $\hat{P}_{I \mid B}$ & Dataset & $\hat{P}_{I \mid B}$ \\
\hline ajaxorange & {$[0.74,0.90]$} & dataminingbook & {$[0.75,0.90]$} \\
apple & {$[0.47,0.67]$} & rapbook & {$[0.40,0.60]$} \\
banana & {$[0.50,0.70]$} & feltflowerrug & {$[0.63,0.81]$} \\
bluescrunge & {$[0.59,0.78]$} & translucentbowl & {$[0.64,0.82]$} \\
dirtyworkgloves & {$[0.55,0.74]$} & greenteabox & {$[0.72,0.88]$} \\
juliespot & {$[0.59,0.78]$} & cardboardbox & {$[0.57,0.76]$} \\
checkeredscarf & {$[0.49,0.69]$} & dirtyrunningshoe & {$[0.27,0.47]$} \\
wd40can & {$[0.73,0.89]$} & largespoon & {$[0.38,0.58]$} \\
candlewithholder & {$[0.71,0.87]$} & goldmedal & {$[0.58,0.77]$} \\
glazedwoodpot & {$[0.45,0.66]$} & spritecan & {$[0.67,0.84]$} \\
cokecan & {$[0.76,0.91]$} & stripednotebook & {$[0.70,0.86]$} \\
smileyfacedoll & {$[0.77,0.91]$} & fabricsoftenerbox & {$[0.69,0.86]$} \\
\hline
\end{tabular}

Table 4 Probabilistic analysis on text data for accuracy. 95\% confidence intervals for the probability that $a_{I}$ is higher, given that $a_{B}$ is higher $\left(\hat{P}_{I \mid B}\right)$.

\begin{tabular}{lc|lc}
\hline Dataset & $\hat{P}_{I \mid B}$ & Dataset & $\hat{P}_{I \mid B}$ \\
\hline alt atheism & {$[0.53,0.73]$} & rec sport hockey & {$[0.77,0.91]$} \\
comp graphics & {$[0.66,0.83]$} & sci crypt & {$[0.56,0.75]$} \\
comp os ms-windows misc & {$[0.53,0.73]$} & sci electronics & {$[0.60,0.79]$} \\
comp sys ibm pc hardware & {$[0.56,0.75]$} & sci med & {$[0.65,0.82]$} \\
comp sys mac hardware & {$[0.63,0.81]$} & sci space & {$[0.70,0.86]$} \\
comp windows x & {$[0.67,0.84]$} & soc religion christian & {$[0.56,0.75]$} \\
misc forsale & {$[0.43,0.63]$} & talk politics guns & {$[0.52,0.72]$} \\
rec autos & {$[0.70,0.87]$} & talk politics mideast & {$[0.62,0.80]$} \\
rec motorcycles & {$[0.65,0.82]$} & talk politics misc & {$[0.39,0.59]$} \\
rec sport baseball & {$[0.64,0.82]$} & talk religion misc & {$[0.44,0.64]$} \\
\hline
\end{tabular}

lowing the usual way of estimating conditional probabilities, $P_{I \mid B}$ is estimated as

$$
\hat{P}_{I \mid B}=\frac{\sum_{C_{1}} \sum_{C_{2} \neq C_{1}} \mathbb{I}\left(a_{I}\left(C_{1}\right)>a_{I}\left(C_{2}\right) \& a_{B}\left(C_{1}\right)>a_{B}\left(C_{2}\right)\right)}{\sum_{C_{1}} \sum_{C_{2} \neq C_{1}} \mathbb{I}\left(a_{B}\left(C_{1}\right)>a_{B}\left(C_{2}\right)\right)},
$$

where the sums range over the different classifiers learned by our 14 learners on the considered dataset, and $\mathbb{I}(c)$ is 1 if $c$ is true and 0 otherwise. Intuitively, $P_{I \mid B}$ indicates how well observations in terms of $a_{B}$ are expected to transfer to $a_{I}$. The converse probability, $P_{B \mid I}=P\left(a_{B}\left(C_{1}\right)>a_{B}\left(C_{2}\right) \mid a_{I}\left(C_{1}\right)>a_{I}\left(C_{2}\right)\right)$, can be estimated similarly. Similar formulas can be used for AUC. Note that when no two classifiers have the same rank, the denominator equals 0.5 and $P_{I \mid B}$ and $P_{B \mid I}$ are identical. In fact, $\hat{P}_{I \mid B}$ and $\hat{P}_{B \mid I}$ are nearly identical for all datasets. Therefore, we only show the confidence intervals for $\hat{P}_{I \mid B}$.

Tables 3, 4, and 5 show the results for accuracy, and Tables 6,7 , and 8 for AUC. Note that if $a_{I}$ and $a_{B}$ were unrelated to each other, we would have $\hat{P}_{I \mid B}=\hat{P}_{B \mid I}=0.5$, while if $a_{I}$ and $a_{B}$ always agreed with each other, we would have $\hat{P}_{I \mid B}=\hat{P}_{B \mid I}=1.0$. This means that the probabilities that we 
Table 5 Probabilistic analysis on UCI data for accuracy. 95\% confidence intervals for the probability that $a_{I}$ is higher, given that $a_{B}$ is higher $\left(\hat{P}_{I \mid B}\right)$.

\begin{tabular}{lc|lc}
\hline Dataset & $\hat{P}_{I \mid B}$ & Dataset & $\hat{P}_{I \mid B}$ \\
\hline Adult 1/2 & {$[0.73,0.89]$} & Spam 2/3 & {$[0.70,0.86]$} \\
Adult 1/3 & {$[0.70,0.86]$} & Spam 1/4 & {$[0.75,0.90]$} \\
Adult 2/3 & {$[0.63,0.81]$} & Spam 2/4 & {$[0.77,0.91]$} \\
Adult 1/4 & {$[0.64,0.82]$} & Spam 1/5 & {$[0.80,0.93]$} \\
Adult 2/4 & {$[0.64,0.82]$} & Spam 2/5 & {$[0.71,0.87]$} \\
Adult 1/5 & {$[0.60,0.79]$} & Spam 1/10 & {$[0.71,0.87]$} \\
Adult 2/5 & {$[0.65,0.83]$} & Spam 2/10 & {$[0.71,0.87]$} \\
Adult 1/10 & {$[0.57,0.76]$} & Tictactoe 1/2 & {$[0.68,0.85]$} \\
Adult 2/10 & {$[0.61,0.80]$} & Tictactoe 1/3 & {$[0.68,0.85]$} \\
Diabetes 1/2 & {$[0.64,0.82]$} & Tictactoe 2/3 & {$[0.72,0.88]$} \\
Diabetes 1/3 & {$[0.49,0.69]$} & Transfusion 1/2 & {$[0.45,0.65]$} \\
Diabetes 2/3 & {$[0.63,0.81]$} & Transfusion 1/3 & {$[0.47,0.67]$} \\
Spam 1/2 & {$[0.77,0.91]$} & Transfusion 2/3 & {$[0.44,0.65]$} \\
Spam 1/3 & {$[0.77,0.91]$} & & \\
\hline
\end{tabular}

Table 6 Probabilistic analysis on SIVAL data for AUC. 95\% confidence intervals for the probability that $\mathrm{AUC}_{I}$ is higher, given that $\mathrm{AUC}_{B}$ is higher $\left(\hat{P}_{I \mid B}\right)$.

\begin{tabular}{lc|lc}
\hline Dataset & $\hat{P}_{I \mid B}$ & Dataset & $\hat{P}_{I \mid B}$ \\
\hline ajaxorange & {$[0.29,0.49]$} & dataminingbook & {$[0.52,0.72]$} \\
apple & {$[0.37,0.57]$} & rapbook & {$[0.57,0.76]$} \\
banana & {$[0.34,0.54]$} & feltflowerrug & {$[0.30,0.50]$} \\
bluescrunge & {$[0.42,0.62]$} & translucentbowl & {$[0.46,0.66]$} \\
dirtyworkgloves & {$[0.37,0.57]$} & greenteabox & {$[0.24,0.43]$} \\
juliespot & {$[0.52,0.72]$} & cardboardbox & {$[0.34,0.54]$} \\
checkeredscarf & {$[0.48,0.68]$} & dirtyrunningshoe & {$[0.45,0.65]$} \\
wd40can & {$[0.19,0.37]$} & largespoon & {$[0.35,0.55]$} \\
candlewithholder & {$[0.37,0.57]$} & goldmedal & {$[0.44,0.64]$} \\
glazedwoodpot & {$[0.47,0.67]$} & spritecan & {$[0.29,0.49]$} \\
cokecan & {$[0.25,0.44]$} & stripednotebook & {$[0.45,0.65]$} \\
smileyfacedoll & {$[0.34,0.54]$} & fabricsoftenerbox & {$[0.15,0.32]$} \\
\hline
\end{tabular}

measured indicate that there is some agreement between $a_{I}$ and $a_{B}$, but it is far from consistent. Choosing the best bag classifier (among two given classifiers) quite often does not yield the best instance classifier, and vice versa. These conclusions are consistent with our previous rank analysis, and quantify the practical implications.

4.3 Experimental analysis of the relationship between bag level and instance level accuracy over multiple datasets

This section addresses the following question: Given a multi-instance algorithm, how do instance-level and bag-level performance correlate with each other over multiple datasets. By studying this relationship for a specific learner, we learn about the effect of the inductive bias of that learner on the correlation between instance- and bag-level performance. 
Table 7 Probabilistic analysis on text data for AUC. 95\% confidence intervals for the probability that $\mathrm{AUC}_{I}$ is higher, given that $\mathrm{AUC}_{B}$ is higher $\left(\hat{P}_{I \mid B}\right)$.

\begin{tabular}{lc|lc}
\hline Dataset & $\hat{P}_{I \mid B}$ & Dataset & $\hat{P}_{I \mid B}$ \\
\hline alt atheism & {$[0.75,0.90]$} & rec sport hockey & {$[0.66,0.83]$} \\
comp graphics & {$[0.56,0.75]$} & sci crypt & {$[0.77,0.91]$} \\
comp os ms-windows misc & {$[0.56,0.75]$} & sci electronics & {$[0.65,0.83]$} \\
comp sys ibm pc hardware & {$[0.64,0.82]$} & sci med & {$[0.77,0.91]$} \\
comp sys mac hardware & {$[0.72,0.88]$} & sci space & {$[0.81,0.94]$} \\
comp windows x & {$[0.82,0.95]$} & soc religion christian & {$[0.73,0.89]$} \\
misc forsale & {$[0.80,0.93]$} & talk politics guns & {$[0.71,0.87]$} \\
rec autos & {$[0.67,0.84]$} & talk politics mideast & {$[0.73,0.89]$} \\
rec motorcycles & {$[0.80,0.93]$} & talk politics misc & {$[0.67,0.84]$} \\
rec sport baseball & {$[0.82,0.95]$} & talk religion misc & {$[0.70,0.86]$} \\
\hline
\end{tabular}

Table 8 Probabilistic analysis on UCI data for AUC. 95\% confidence intervals for the probability that $\mathrm{AUC}_{I}$ is higher, given that $\operatorname{AUC}_{B}$ is higher $\left(\hat{P}_{I \mid B}\right)$.

\begin{tabular}{lc|lc}
\hline Dataset & $\hat{P}_{I \mid B}$ & Dataset & $\hat{P}_{I \mid B}$ \\
\hline Adult 1/2 & {$[0.81,0.94]$} & Spam 2/3 & {$[0.72,0.88]$} \\
Adult 1/3 & {$[0.71,0.87]$} & Spam 1/4 & {$[0.61,0.80]$} \\
Adult 2/3 & {$[0.80,0.93]$} & Spam 2/4 & {$[0.61,0.80]$} \\
Adult 1/4 & {$[0.76,0.91]$} & Spam 1/5 & {$[0.58,0.77]$} \\
Adult 2/4 & {$[0.78,0.92]$} & Spam 2/5 & {$[0.59,0.78]$} \\
Adult 1/5 & {$[0.73,0.89]$} & Spam 1/10 & {$[0.57,0.76]$} \\
Adult 2/5 & {$[0.72,0.88]$} & Spam 2/10 & {$[0.51,0.71]$} \\
Adult 1/10 & {$[0.63,0.81]$} & Tictactoe 1/2 & {$[0.78,0.92]$} \\
Adult 2/10 & {$[0.64,0.82]$} & Tictactoe 1/3 & {$[0.76,0.91]$} \\
Diabetes 1/2 & {$[0.86,0.97]$} & Tictactoe 2/3 & {$[0.86,0.97]$} \\
Diabetes 1/3 & {$[0.72,0.88]$} & Transfusion 1/2 & {$[0.80,0.93]$} \\
Diabetes 2/3 & {$[0.76,0.91]$} & Transfusion 1/3 & {$[0.75,0.90]$} \\
Spam 1/2 & {$[0.75,0.90]$} & Transfusion 2/3 & {$[0.80,0.93]$} \\
Spam 1/3 & {$[0.68,0.85]$} & & \\
\hline
\end{tabular}

\subsubsection{Algorithms}

We first briefly introduce how each algorithm learns a classifier. We partition the algorithms in two groups, inspired by the taxonomies introduced by Amores (2013) and Foulds and Frank (2010).

The first group of algorithms, which we will call standard MI algorithms, learn an instance classifier $f$ and follow the standard MI assumption, meaning they learn an instance classifier that classifies a bag as positive if at least one of the instances is classified as positive. No further information about the properties of the bag as a whole is used. This group consists of the following seven algorithms: MIDD, MIEMDD, MISVM, MIOptimalBall, MILR, MIRI, and AdaBoost.M1-MITI.

The remaining seven algorithms depart from the standard MI assumption in different ways. While different subcategories can be defined to group them, for our purposes it is sufficient to label them as non-standard MI algorithms. MIWrapper-J48 transforms the multi-instance problem into a single-instance 
problem by applying the bag label to every instance. It assumes that every instance in a bag contributes equally to the bag label, contrary to the standard MI assumption, where a single positive instance makes a bag positive. SimpleMI-J48, MILRC, and MDD map the instances in a bag to a single feature vector by averaging each feature over these instances. Learning a classifier is based on aggregate information about a bag, instead of on the individual instances. Finally, Citation-kNN, MISMO, and TLD use knowledge about the bag as a whole to learn a classifier. Citation-kNN and MISMO use respectively a distance or kernel function defined over the bag, and TLD learns a classifier from both the distributional properties of the data on the instance level as well as those on the bag level.

While this work focuses on the standard MI problem, we also included algorithms that do not follow this assumption. One might argue that these algorithms are not designed to classify instances. However, a distinction should be made between the problem definition (standard MI learning) and the solving technique (the learner). As our experiments show, some of the non-standard MI algorithms are indeed suitable for classifying instances and sometimes even outperform the standard MI algorithms on this task. An additional argument for including these algorithms is that researchers may not always be aware of the assumptions an algorithm makes and compare different types of algorithms. Our experiments thus reflect a realistic situation.

\subsubsection{Results}

The theoretical relationship between $a_{I}$ and $a_{B}$ was demonstrated in Figure 1 in section 2.4 with a scatter plot showing $a_{I}$ and $a_{B}$ on a set of random artificial MI problems. We now experimentally construct similar scatter plots for each of our fourteen algorithms, indicating for each individual result the category of the dataset: SIVAL (red square), text (green circle), or UCI (blue triangle).

Figure 10 shows the results measured in accuracy and Figure 11 in AUC. We summarize our most important findings.

First, we see that the domain of the dataset is an important factor for determining both instance- and bag-level performance. In all figures, the results are clearly clustered according to category.

Second, we see that, despite assuming the existence of instance labels, standard MI algorithms do not always have good instance-level performance. Inspecting the individual predictions for these algorithms showed that they predict almost all negative instances correctly, but often fail for the positive instances. It has been observed before by Liu et al (2012) that, while these algorithms assign instance labels, they focus on maximizing bag-level performance. The classifiers are thus good at identifying the most positive example in a bag, but not at finding all positive instances in a bag. However, since in our datasets the majority of instances are negative, bad performance on the positive instances has little effect on the accuracy. This is visible in the scatter plots for accuracy in Figure 10: For the text datasets, the standard MI algorithm $a_{I}$ is always close to one, while $a_{B}$ shows variation. As a result, 
bag-level and instance-level accuracy should not be substituted for each other for these algorithms.

The ability of an algorithm to classify positive instances is judged better on the basis of the AUC scatter plots in Figure 11. For instance, Diverse Density (MIEMDD, MIDD and MDD) and OptimalBall have a low $\mathrm{AUC}_{I}$ on almost all of the text and SIVAL datasets. These algorithms explicitly assume that all positive instances lie close to a single point in the instance space, determined by maximizing bag-level accuracy through an optimization procedure. The low $\mathrm{AUC}_{I}$ indicates that this assumption does not work well here. However, the classifiers often detect at least one positive instance in the positive bags, demonstrated by the overall high $\mathrm{AUC}_{B}$ on the datasets. Conversely, AdaBoost.M1-MITI and MIRI naturally learn disjunctive concepts and are therefore better at identifying multiple positive instances in a bag, resulting in a higher $\mathrm{AUC}_{I}$ and therefore also a stronger correspondence between $\mathrm{AUC}_{I}$ and $\mathrm{AUC}_{B}$.

A third observation is that accuracy is not always the most appropriate performance measure for multi-instance problems. Furthermore, the performance measure influences the correspondence between instance- and bag-level performance. Multi-instance problems often have a highly skewed class distribution, and accuracy therefore does not give enough weight to the correct classification of positive instances, while identifying the characteristics of the positive instances is essential in standard MI learning. This is not always problematic, however: The UCI datasets for instance do not have large class skew so they do not exhibit the described behavior.

We focus the remaining part of our discussion on the non-standard MI algorithms. Since these algorithms do not adhere to the standard MI assumption, one might expect that they will be worse at classifying instances.

An example is MISMO on the SIVAL datasets. It can be seen from Figure 10 that instance-level accuracy varies over a wide range. MISMO tends to either predict all instances in the test set to be positive, or negative. This disagreement between instance- and bag-level accuracy of MISMO has been noted before by Doran and Ray (2013), who showed that the set kernel used by MISMO can separate bags even when the corresponding instance kernel cannot separate the instances. It seems that for the SIVAL datasets, the classifier has learned to separate bags based on overall properties of the bags, whereas, judging from the higher $\mathrm{AUC}_{I}$, for the text and UCI datasets individual properties of the instances are used. This again demonstrates the importance of the characteristics of the datasets in the relationship between instance- and bag-level performance.

A non-standard MI algorithm that can classify instances well is Citation$\mathrm{kNN}$. The scatter plot shows that on most UCI and SIVAL datasets, $\mathrm{AUC}_{I}$ is higher than $\mathrm{AUC}_{B}$.

Finally, we observe that the distance based approaches, i.e., Citation-kNN, MIOptimalBall and the Diverse Density variants, do not perform well on the text datasets in terms of either bag- and instance-level AUC. For MDD and MIEMDD, the AUC for the text datasets is even smaller than 0.5. By in- 
specting the individual predictions, we saw that not a single positive instance is classified positive, whereas a small number of negative instances are incorrectly classified as positive. This is explained by the presence of many irrelevant attributes (Dooly et al, 2003), and the high dimensionality of the data, which makes distance less effective as a measure of similarity.

From this discussion we conclude that the relationship between instanceand bag-level performance is determined by the domain of the dataset, the learner assumptions, and the performance measure. However, even if the correlation between, for instance, $a_{I}$ and $a_{B}$, were perfectly linear for each classifier, the regression slope could still differ, resulting again in different rankings of classifiers in terms of instance- and bag-level accuracy.

Note that these results are consistent with the results in Section 4.2.1. For instance, we see in Figure 10(7) that AdaBoost.M1 consistently has better $a_{I}$ than $a_{B}$ on the SIVAL datasets, and that this is again observed in the rankings of the fourteen learners on the separate datasets in Figure 4.

\section{Comparison of multi-instance and single-instance learning algorithms}

In the multi-instance literature, experimental studies have mostly been conducted in terms of bag-level performance (Dietterich et al, 1997; Maron and Lozano-Pérez, 1998; Zhang and Goldman, 2001; Wang and Zucker, 2000; Blockeel et al, 2005; Ramon and De Raedt, 2000; Zhou and Zhang, 2003; Xu and Frank, 2004; Zucker and Chevaleyre, 2001; Andrews et al, 2003; Ray and Craven, 2005; Tao et al, 2004; Fung et al, 2007). Now that we have established that the correlation between instance-level and bag-level performance is weak, the question is whether the conclusions from these studies also apply in terms of instance-level performance or not. Below we investigate one particular aspect of this question, namely: when evaluated in terms of instance-level performance, do multi-instance learning algorithms indeed perform better on multi-instance problems than single-instance learning algorithms do? Like Ray and Craven (2005), we limit our study to AUC.

The context of this question is that multi-instance (MI) learning can be tackled with single-instance (SI) algorithms: simply label each instance with the label of its bag and apply a standard SI learning algorithm to the resulting dataset. Many instances will have a positive label in the dataset even if they are really negative. The opposite will not occur, so we get a dataset with one-sided class noise. The question is then how well SI learning performs when applied to this dataset, as compared to MI learning applied to the original MI dataset. This has been investigated in the literature, with mixed results: Dietterich et al (1997) found the SI approach not to work well, while Ray and Craven (2005) provide a more complex picture, showing that this may depend strongly on the data and the actual learner used. Both papers measured performance on the bag-level only. In this paper, we investigate the same question, but in terms of instance-level performance. 


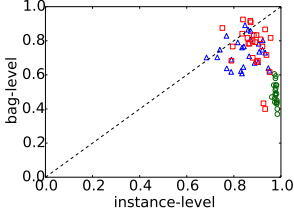

(1) MIDD

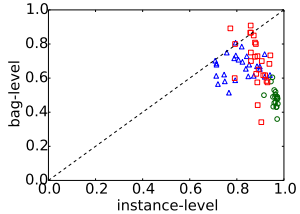

(4) MIOptimalBall

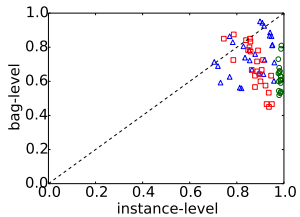

(7) AdaBoost.M1

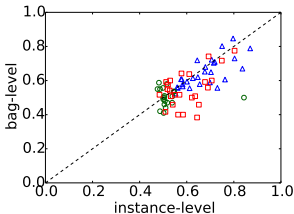

(10) MILRC

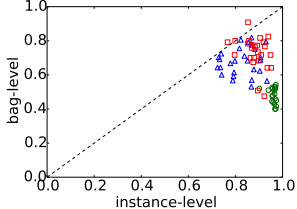

(2) MIEMDD

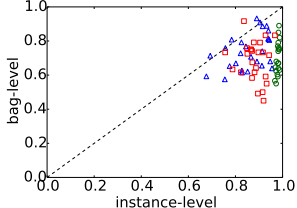

(5) MILR

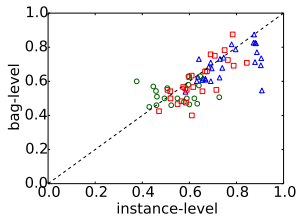

(8) MIWrapper

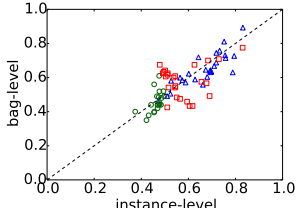

(11) MDD

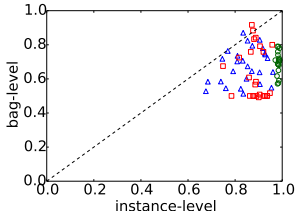

(3) MISVM

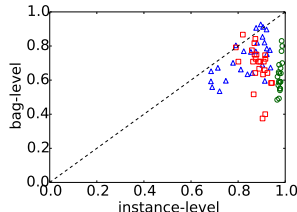

(6) MIRI

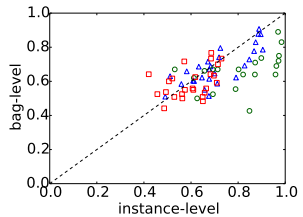

(9) SimpleMI

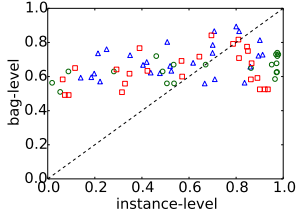

(12) MISMO

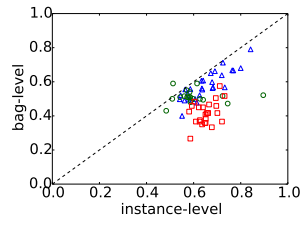

(13) CitationKNN

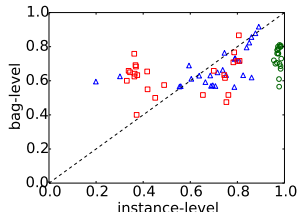

(14) TLD

Fig. 10 Scatter plots showing bag-level accuracy versus instance-level accuracy for each classifier over the semi-synthetic (blue triangle), newsgroup (green circle), and SIVAL (red square) datasets.

The experimental setup is similar to that of the previous section, although here we only use a selection of six MI learning algorithms that are based on some SI algorithm. These six algorithm pairs are listed in Table 9. On each dataset, we compare the instance-level AUC of each MI algorithm with that of its corresponding SI algorithm. A scatter plot is constructed for each pair of learners, plotting the SI and MI AUC of the learner on each dataset. The results are shown in Figure 12. 


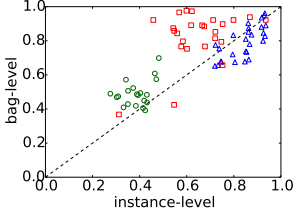

(1) MIDD

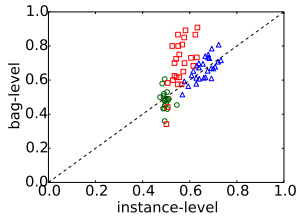

(4) MIOptimalBall

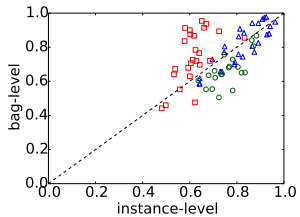

(7) AdaBoost.M1

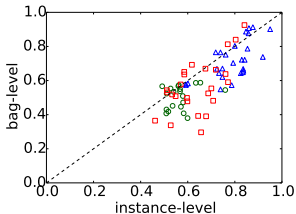

(10) MILRC

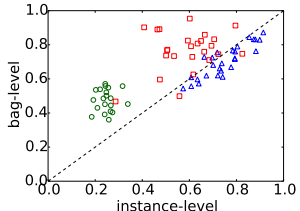

(2) MIEMDD

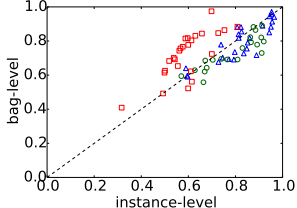

(5) MILR

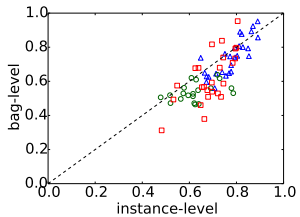

(8) MIWrapper

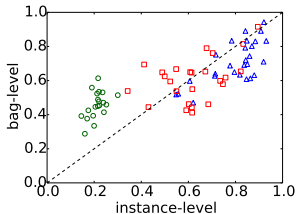

(11) MDD

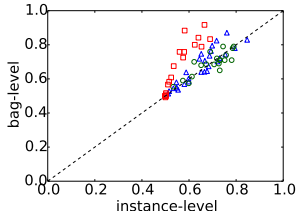

(3) MISVM

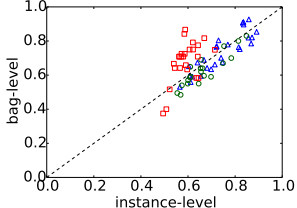

(6) MIRI

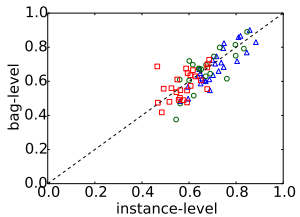

(9) SimpleMI

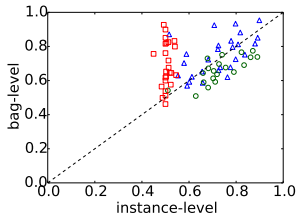

(12) MISMO

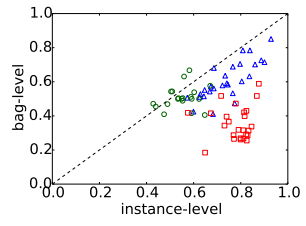

(13) CitationKNN

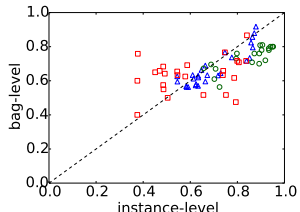

(14) TLD

Fig. 11 Scatter plots showing bag-level AUC versus instance-level AUC for each classifier over the semi-synthetic (blue triangle), newsgroup (green circle), and SIVAL (red square) datasets.

Our results show that in many cases the MI algorithms do not clearly outperform their SI counterparts. This is for instance the case for SimpleMI-J48 and MIWrapper. Both these algorithms are based on the application of J48 on a single-instance dataset derived from the original multi-instance dataset. SimpleMI does this by averaging the features over all instances in a bag. MIWrapper, similar to our own conversion of the multi-instance datasets, assigns the bag label to each instance in the bag, after which it also reweighs the instances so that each bag has the same weight independent of the number 
Table 9 Multi-instance (MI) algorithms and their corresponding single-instance (SI) algorithm.

\begin{tabular}{ll}
\hline MI algorithm & SI algorithm \\
\hline MIWrapper-J48 (Frank and Xu, 2003) & J48 (Quinlan, 2003) \\
SimpleMI-J48 (Dong, 2006) & J48 (Quinlan, 2003) \\
Citation-kNN (Wang and Zucker, 2000) & kNN (Aha et al, 1991) \\
MILR (Ray and Craven, 2005) & Logistic (le Cessie and van Houwelingen, 1992) \\
MISMO (Xu, 2003) & SMO (Platt, 1999) \\
AdaBoost.M1-MITI & AdaBoost.M1-J48 \\
(Freund and Schapire, 1995; Blockeel et al, 2005) & (Freund and Schapire, 1995; Quinlan, 2003) \\
\hline
\end{tabular}

of instances in it. However, in our experiments this extra step did not influence the performance much; except for the text datasets all bags have more or less the same size. Consequently, the difference between the performance of MIWrapper-J48 and J48 is very small. Moreover, for the text datasets, which have varying bag sizes, the reweighing does not always lead to improved performance of MIWrapper-J48.

SimpleMI-J48 outperforms J48 only for the text datasets. We already saw in Section 4.3.2 that SimpleMI-J48 has good performance for the text datasets, both for bag- and instance-level classification. The fact that the text data has many irrelevant attributes with a value close to zero, explains why averaging works well here.

AdaBoost.M1-MITI clearly outperforms J48 for the text datasets, and it has a slight advantage on the UCI data. On the SIVAL datasets, AdaBoost.M1J48 mostly wins against AdaBoost.M1-MITI.

There is almost no difference between the performance of Citation-kNN and single-instance kNN. With only a single instance in a test bag, identifying references with the minimal Hausdorff distance is the same as finding the nearest neighbor in the training set. Furthermore, the results show that the closest citations are mostly identical to the closest references and using them does not make a big difference in performance.

Lastly, MI-Logistic regression (MILR) often wins against its single-instance variant for the text and UCI datasets The same goes for MISMO, which we also observed not to work well for instance-level classification on the SIVAL datasets.

These results supplement Ray and Craven's conclusions, showing again a mixed picture. Whether an MI learner outperforms its SI counterpart depends on the domain of the dataset in combination with the type of learner. It happens frequently that an SI learner performs almost as well as an MI learner, or even outperforms it.

\section{Conclusions}

In the current literature on multi-instance learning, almost all empirical evaluations use bag-level classification performance. However, in a multi-instance 


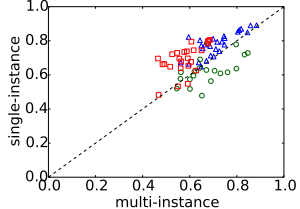

(1) SimpleMI-J48

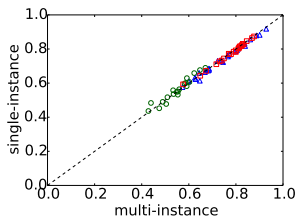

(4) CitationkNN-kNN

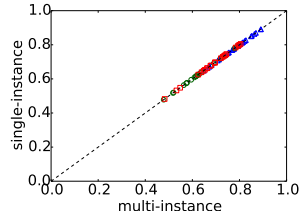

(2) MIWrapper-J48

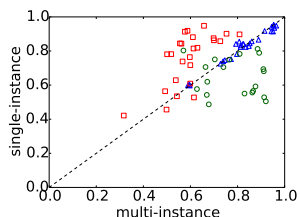

(5) MILR-Logistic

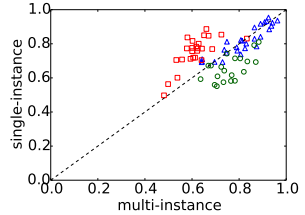

(3) AB-MITI-AB-J48

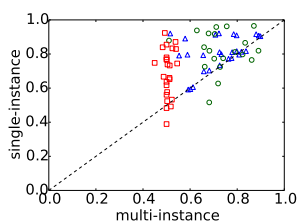

(6) MISMO-SMO

Fig. 12 Instance-level AUC of single-instance versus multi-instance algorithms. ('AB' stands for AdaBoost.M1)

setting, the task of classifying instances is often interesting by itself as well. However, very little is known about how well methods typically perform on this task, and to what extent conclusions of experimental studies in terms of bag-level performance can be generalized to the instance-level. The aim of this paper was to investigate this.

First, we examined the correlation between instance-level and bag-level performance of a set of classifiers, when ranking them according to one or the other. We find that this correlation is relatively weak, both in terms of accuracy and AUC. The "best" bag classifiers do not necessarily yield the best (or even good) instance classifiers. Our experiments are consistent with our theoretical analysis, and additionally demonstrate that the strength of the correlation varies with the specific problem, i.e., the domain of the dataset and the chosen performance measure.

Second, we examined the relationship between instance-level and bag-level performance of a single classifier over multiple datasets. We again found a mixed picture where the correspondence between instance- and bag-level performance of a learner on a give dataset depends on the the data domain, the performance measure (accuracy or AUC), and the learner in question. These results provide some explanation for the difference in rankings of a set of classifiers in terms of bag- and instance-level performance.

Finally, we also investigated whether multi-instance learning algorithms perform better than their single-instance counterparts (on one-sided noisy data) in terms of instance-level AUC. We found that single-instance algorithms often outperform multi-instance algorithms, but this again depends on the specific problem.

Given the relatively weak correlation between bag-level and instance-level performance, we conclude that it is advisable for researchers proposing novel multi-instance algorithms to test their methods on both levels, or to state explicitly which level is of interest to them, and evaluate according to that 
level. Moreover, our experimental results showed that multi-instance classifiers are very sensitive to the context in which they are used, and this should be taken into account when evaluating multi-instance algorithms.

\section{Acknowledgements}

Research supported by Research Foundation Flanders (FWO-Vlaanderen) projects G.0413.09 and G.0682.11, and by the Research Fund KU Leuven (GOA/08/008).

\section{References}

Aha D (1990) Incremental constructive induction: An instance-based approach. In: Proceedings of the 7th International Conference on Machine Learning, Morgan Kaufmann, pp 117-121

Aha D, Kibler D, Albert M (1991) Instance-based learning algorithms. Machine learning 6(1):37-66

Amores J (2013) Multiple instance classification: Review, taxonomy and comparative study. Artificial Intelligence 201:81-105

Andrews S, Tsochantaridis I, Hofmann T (2003) Support vector machines for multiple-instance learning. In: Advances in Neural Information Processing Systems 15 (NIPS), MIT Press, pp 577-584

Auer P, Ortner R (2004) A boosting approach to multiple instance learning. In: Proceedings of the 15th European Conference on Machine Learning, Lecture Notes in Computer Science, vol 3201, Springer, pp 63-74

Auer P, Long P, Srinivasan A (1998) Approximating hyper-rectangles: learning and pseudo-random sets. Journal of Computer and System Sciences $57(3): 376-388$

Bjerring L, Frank E (2011) Beyond trees: Adopting miti to learn rules and ensemble classifiers for multi-instance data. In: Proceedings of the 24th Australian Joint Conference on Artificial Intelligence, Springer, Perth, Australia, pp 41-50

Blockeel H, Page D, Srinivasan A (2005) Multi-instance tree learning. In: Proceedings of the 22d International Conference on Machine learning, ACM Press, pp 57-64

Blum A, Kalai A (1998) A note on learning from multiple-instance examples. Machine Learning 30(1):23-29

le Cessie S, van Houwelingen J (1992) Ridge estimators in logistic regression. Applied Statistics 41(1):191-201

Chen Y, Wang J (2004) Image categorization by learning and reasoning with regions. Journal of Machine Learning Research 5:913-939

Cranor L, LaMacchia B (1998) Spam! Communications of the ACM 41(8):7483

Dietterich T, Lathrop R, Lozano-Pérez T (1997) Solving the multiple instance problem with axis-parallel rectangles. Artificial Intelligence 89(1-2):31-71 
Dong L (2006) A comparison of multi-instance learning algorithms. Master's thesis

Dooly D, Zhang Q, Goldman S, Amar R (2003) Multiple instance learning of real valued data. Journal of Machine Learning Research 3:651-678

Doran G, Ray S (2013) A theoretical and empirical analysis of support vector machine methods for multiple-instance classification. Machine Learning pp $1-24$

Foulds J, Frank E (2010) A Review of Multi-Instance Learning Assumptions. Knowledge Engineering Review 25:1-25

Frank E, Xu X (2003) Applying propositional learning algorithms to multiinstance data. Tech. rep., University of Waikato

Freund Y, Schapire R (1995) A decision-theoretic generalization of on-line learning and an application to boosting. In: Proceedings of the 2d European Conference on Computational Learning Theory, Springer-Verlag, pp 23-37

Fu Z, Robles-Kelly A, Zhou J (2011) MILIS: multiple instance learning with instance selection. IEEE Transactions on Pattern Analysis and Machine Intelligence 33(5):958-977

Fung G, Dundar M, Krishnapuram B, Rao R (2007) Multiple instance learning for computer aided diagnosis. In: Advances in Neural Information Processing Systems 19 (NIPS), MIT Press, pp 425-432

Gärtner T, Flach P, Kowalczyk A, Smola A (2002) Multi-instance kernels. In: Proceedings of the 19th International Conference on Machine Learning, Morgan Kaufmann, pp 179-186

Kohavi R (1996) Scaling up the accuracy of naive-Bayes classifiers: A decisiontree hybrid. In: Proceedings of the 2d International Conference on Knowledge Discovery and Data Mining, AAAI Press, vol 7, pp 202-207

Li Y, Kwok J, Tsang I, Zhou Z (2009) A convex method for locating regions of interest with multi-instance learning. In: Proceedings of the European Conference on Machine Learning and Knowledge Discovery in Databases, Springer-Verlag, pp 15-30

Liu G, Wu J, Zhou Z (2012) Key instance detection in multi-instance learning. In: Hoi SCH, Buntine WL (eds) Proceedings of the 4th Asian Conference on Machine Learning, JMLR.org, JMLR Proceedings, vol 25, pp 253-268

Long P, Tan L (1998) PAC learning axis-aligned rectangles with respect to product distributions from multiple-instance examples. Machine Learning 30(1):7-21

Mandel M, Ellis D (2008) Multiple-instance learning for music information retrieval. In: Proceedings of the 9th International Conference on Music Information Retrieval, pp 577-582

Maron O, Lozano-Pérez T (1998) A framework for multiple-instance learning. In: Advances in Neural Information Processing Systems 11 (NIPS), MIT Press, pp 570-576

Maron O, Ratan A (1998) Multiple-instance learning for natural scene classification. In: Proceedings of the 15th International Conference on Machine Learning, Morgan Kaufmann, pp 341-349 
Merz C, Murphy P (1996) UCI repository of machine learning databases. Http://archive.ics.uci.edu/ml/

Tragante do O V, Fierens D, Blockeel H (2011) Instance-level accuracy versus bag-level accuracy in multi-instance learning. In: Proceedings of the 23d Benelux Conference on Artificial Intelligence, URL https://lirias. kuleuven. be/handle/123456789/316681

Platt J (1999) Fast training of support vector machines using sequential minimal optimization. Advances in Kernel Methods-Support Vector Learning 208:185-208

Quinlan J (2003) C4.5: programs for machine learning. Morgan Kaufmann

Ramon J, De Raedt L (2000) Multi instance neural networks. In: Proceedings of the 17th International Conference on Machine Learning, Workshop on Attribute-Value and Relational Learning, pp 53-60

Ray S, Craven M (2005) Supervised versus multiple instance learning: An empirical comparison. In: Proceedings of the 22d International Conference on Machine Learning, ACM Press, vol 22, pp 697-704

Ray S, Scott S, Blockeel H (2011) Multi-instance learning. Encyclopedia of Machine Learning, first edition pp 701-710

Settles B, Craven M, Ray S (2008) Multiple-instance active learning. In: Advances in Neural Information Processing Systems 20 (NIPS), MIT Press, pp 1289-1296

Shao J, He D, Yang Q (2008) Multi-semantic scene classification based on region of interest. In: Proceedings of the International Conference on Computational Intelligence for Modelling Control \& Automation, IEEE Computer Society, Washington, DC, USA, pp 732-737

Smith J, Everhart J, Dickson W, Knowler W, Johannes R (1988) Using the ADAP learning algorithm to forecast the onset of diabetes mellitus. In: Proceedings of the Symposium on Computer Applications and Medical Care, pp 261-265

Tao Q, Scott S, Vinodchandran N, Osugi T (2004) SVM-based generalized multiple-instance learning via approximate box counting. In: Proceedings of the 21th International Conference on Machine learning, Morgan Kaufmann

Wang J, Zucker J (2000) Solving the multiple-instance problem: A lazy learning approach. In: Proceedings of the 17th International Conference on Machine Learning, Morgan Kaufmann, pp 1119-1126

Witten I, Frank E (2005) Data Mining: Practical machine learning tools and techniques

Xu X (2003) Statistical learning in multiple instance problems. Master's thesis

Xu X, Frank E (2004) Logistic regression and boosting for labeled bags of instances. In: Proceedings of the Pacific Asia Conference on Knowledge Discovery and Data Mining, Lecture Notes in Computer Science, Springer, pp 272-281

Yeh I, Yang K, Ting T (2009) Knowledge discovery on RFM model using Bernoulli sequence. Expert Systems With Applications 36(3P2):5866-5871

Zhang M (2009) Generalized multi-instance learning: Problems, algorithms and data sets. In: Proceedings of the WRI Global Congress on Intelligent 
Systems, Morgan Kaufmann, vol 3, pp 539-543

Zhang Q, Goldman S (2001) EM-DD: An improved multiple-instance learning technique. In: Advances in Neural Information Processing Systems 14 (NIPS), MIT Press, pp 1073-1080

Zhou Z, Zhang M (2003) Ensembles of multi-instance learners. In: Proceedings of the 14th European Conference on Machine Learning, Lecture Notes in Computer Science, Springer, pp 492-502

Zhou Z, Xue X, Jiang Y (2005) Locating regions of interest in cbir with multiinstance learning techniques. In: Proceedings of the 18th Australian Joint conference on Advances in Artificial Intelligence, Springer-Verlag, pp 92-101

Zucker J, Chevaleyre Y (2001) Solving multiple-instance and multiple-part learning problems with decision trees and decision rules. Application to the mutagenesis problem. In: Proceedings of the 14th Biennial Conference of the Canadian Society on Computational Studies of Intelligence: Advances in Artificial Intelligence, Springer, pp 204-214 